\title{
Planeamiento Integrado de la Expansión en Generación y Transmisión Considerando Restricciones de Seguridad
}

\author{
Sergio D. Saldarriaga-Zuluaga, Jesús M. López-Lezama y Nicolás Muñoz-Galeano \\ Fac. de Ingeniería, Dpto. Ing. Eléctrica, Grupo de Manejo Eficiente de la Energía - GIMEL, Universidad de \\ Antioquia, Calle 67 No. 53-108, Oficina 19-437, Medellín, Colombia \\ (e-mail: sergio.saldarriaga@udea.edu.co,jmaria.lopez@udea.edu.co,nicolas.munoz@udea.edu.co)
}

Recibido Ago. 24, 2017; Aceptado Nov. 13, 2017; Versión final Dic. 12, 2017, Publicado Jun. 2018

\section{Resumen}

Este artículo presenta un modelo para el Planeamiento Integrado de la Expansión en Generación y Transmisión (PIEGT) considerando restricciones de seguridad. El modelo propuesto combina el problema de planeamiento de expansión de la transmisión (PET) con el problema de planeamiento de la expansión en generación (PEG) adicionando restricciones de seguridad (criterio N-1). El problema resultante (PIEGT) es abordado usando un modelo de programación lineal entera mixta. La principal contribución del artículo es la introducción de restricciones de seguridad en el PIEGT. Los resultados muestran que incluir generación a pequeña escala en el planeamiento de redes de transmisión reduce el número de líneas y los costos totales en los se incurre para atender la demanda esperada. Se realizaron pruebas utilizando el sistema Garver de 6 barras y los sistemas IEEE de 24 y 48 barras, evidenciando la aplicabilidad del modelo propuesto.

\section{Integrated Transmission and Generation Expansion Planning considering Safety Constraints}

\begin{abstract}
This paper presents a model for the integrated Transmission and Generation Expansion Planning (TGEP) considering security constraints. The proposed model combines the transmission network expansion (TNE) planning problem with the generation expansion planning (GEP) problem considering security constraints ( $N$ 1 criteria). The resulting problem (TGEP) is approached by means of a mixed integer linear programming model. The main contribution of the paper is the inclusion of security constraints in the TGEP. Results show that including small scale generation in the transmission network expansion planning reduces the number of lines and associated costs incurred in attending the expected demand. Tests were performed with the 6 bus Garver system as well as the IEEE 24 and 48 bus power systems showing the applicability of the proposed model.
\end{abstract}




\section{INTRODUCCIÓN}

Tradicionalmente el ejercicio de planeación de sistemas de potencia se ha dividido en dos etapas o problemas separados: Planeamiento de la Expansión en Generación (PEG) y Planeamiento de la Expansión en Transmisión (PET). El primero se encarga de determinar la entrada de nuevas fuentes de generación y se hace desde una perspectiva energética (no se consideran las restricciones de la red de transporte). Por otro lado, dados los puntos de suministro identificados en el PEG, el PET se encarga de determinar los nuevos refuerzos de red necesarios para garantizar el abastecimiento de la demanda.

Según Alizadeh y Jadid (2011) dos razones dan cuenta de la separación de estos problemas. En primer lugar, más del $80 \%$ del costo total de la planeación es atribuible al PEG. De esta forma, resolver primero el PEG y usar esta solución como punto de partida para el PET no causa una desviación considerable de la solución óptima del problema combinado. Sin embargo, si solucionar el problema integrado resulta en un porcentaje pequeño de ahorro con respecto a solucionar ambos problemas por separado, este valor sería importante dada la enorme cantidad de dinero asociada al planeamiento del sistema. En segundo lugar, tanto el PEG como el PET son problemas de optimización no convexos que involucran variables de decisión de naturaleza continua y entera. Si el costo computacional de solucionar cualquiera de estos problemas, por separado, ya es considerable, abordar el problema del planeamiento integrado de la expansión en generación y transmisión (PIEGT) de forma estricta requiere de tanto esfuerzo computacional que el tiempo de solución puede resultar, en ocasiones, prohibitivo. Sin embargo, los avances tecnológicos en computación junto con el uso de modelos compactos y métodos de descomposición han contribuido a plantear la solución del PIEGT con tiempos de cómputo aceptables.

Dada la importancia que tiene la electricidad en las sociedades modernas, en la literatura técnica se han reportado numerosos estudios que abordan el PET y el PEG, tanto de forma separada como conjunta. Una búsqueda en las principales bases de datos da cuenta de un par de miles de publicaciones al respecto. Este número elevado de publicaciones se debe principalmente a variaciones en el abordaje del problema, principalmente con respecto a detalles de modelado y técnicas de solución. Otras variaciones incluyen la consideración o no de incertidumbre, integración de renovables, incorporación de criterios de seguridad y confiabilidad.

El objetivo principal del PET es minimizar el costo de los refuerzos de la red; sin embargo, puede tener en cuenta otros aspectos como facilitar la competencia entre los agentes del mercado (Alizadeh y Jadid, 2011), aliviar la congestión de la red de transporte (Akbari et al., 2011), mejorar la confiabilidad (Majidi y Baldick, 2016) y minimizar riesgos económicos (Hobbs et al., 2016). A diferencia del PEG, el PET considera la red de transmisión. Existen básicamente dos formas de modelar la red: modelo AC y modelo DC. Si bien el modelo AC es más aproximado a la operación real, este puede traer desventajas desde el punto de vista de complejidad matemática al introducir no linealidades que pueden evitarse con el modelo DC (Tejada et al., 2014). Usar un modelo AC de la red para el PET puede tener valor práctico cuando se considera un horizonte de planeación de corto plazo. Esto se debe a que la principal diferencia con el modelo DC es la consideración de límites en reactivos y magnitudes de tensión. Estos problemas son abordados normalmente en la operación y no en la planeación, pues son localizados y de corto plazo. En (Macedo et al., 2016; Gomes y Saraiva, 2016) se presentan algunos estudios del PET que usan el modelo AC de la red de transmisión. Una lectura más profunda de las variantes del PET, su clasificación detallada y análisis se puede consultar en las revisiones bibliográficas realizadas en (Hemmati y Hooshmand, 2013; Quintero et al., 2014).

Cuando el planeamiento de la expansión en generación y transmisión se aborda de forma separada primero se soluciona el PEG y su solución sirve como punto de partida para el PET. Así pues, el PEG determina los puntos de suministro y el PET los refuerzos de red. Existe una gran cantidad de variantes con respecto a detalles de modelado y métodos de solución que han sido propuestos para el PEG. Si bien minimizar el costo de instalación y operación de las nuevas unidades generadoras es el principal objetivo del PEG, también se han considerado aspectos como la maximización de las ganancias de los generadores en mercados desregulados (Cerda et al., 2011), maximización de la confiabilidad (Murugan et al.; 2009) y minimización de emisiones contaminantes (Tekiner et al., 2010). Estudios recientes sobre el PEG han prestado especial atención al tema ambiental. Por ejemplo, en (Saboori y Reza, 2016), se aborda el PEG considerando captura de carbono; mientras en (Bent y Toole, 2012), se analizan alternativas de expansión en generación buscando la reducción de emisiones de CO2. La literatura técnica sobre el PEG es abundante. Una lectura más detallada sobre el PEG y sus variantes con respecto a modelado y técnicas de solución se puede consultar en (Phupha y Lantharthong, 2012).

El planeamiento integrado, como su nombre lo indica, consiste en agrupar en un solo problema la expansión de la generación y la transmisión. EI PIEGT combina los desafíos del PEG y PET: no linealidad, no convexidad, incertidumbre y alta dimensionalidad. En la última década, diversos estudios han abordado este 
problema considerando diferentes modelos, métodos y funciones objetivo. Los métodos de solución aplicados al PEG y PET también han sido aplicados al PIEGT. Por ejemplo, en (Pozo et al., 2013; Benidris et al., 2016), se utilizan métodos de programación matemática clásica para abordar este problema mientras en (Saldarriaga et al., 2016a), se utiliza un método metaheurístico. Uno de los aspectos recurrentes en las investigaciones actuales sobre el PIEGT es la integración de renovables; por ejemplo, en (Li et al., 2016), se propone un modelo de planeamiento integrado considerando generación eólica y respuesta de la demanda, mientras en (Ramírez et al., 2016), se plantea un problema de co-optimización de expansión en generación considerando la masificación de vehículos eléctricos. En (Rouhani et al., 2014), se considera un modelo de planeamiento para integrar generación distribuida. En (Saldarriaga et al., 2016b), se presenta un artículo de revisión donde se pueden consultar con mayor detalle los diferentes aspectos relacionados con el PIEGT.

En este artículo se propone un modelo matemático basado en programación lineal entera mixta para realizar el planeamiento integrado de generación- transmisión en sistemas de potencia. El aporte principal consiste en la incorporación de restricciones de seguridad (criterio N-1), que si bien se han tenido en cuenta en modelos aplicados al PET no se han considerado en el PIEGT. Este tipo de modelos contribuyen a la discusión sobre integración de problemas PET-PEG haciendo énfasis en la inclusión de generación pequeña escala y pueden servir de base para discusiones posteriores sobre viabilidad de proyectos de generación distribuida e integración de renovables en el planeamiento de los sistemas de potencia. Los resultados en sistemas de prueba de 6, 24 y 48 barras evidencian la aplicabilidad del modelo propuesto. Se pudo verificar que incluir generación a pequeña escala en el planeamiento de la transmisión conduce a reducción en el costo del planeamiento, pues es posible evitar o aplazar la construcción de algunas líneas de transmisión.

\section{FORMULACIÓN MATEMÁTICA}

La formulación matemática del PIEGT considerando restricciones de seguridad se presenta en las ecuaciones (1)-(19). El objetivo consiste en minimizar los costos de operación y de inversión de la expansión, considerando contingencias simples en la red (criterio $\mathrm{N}-1$ ) incorporadas al modelo por medio de restricciones.

$$
\text { Min: } f 1=\sum_{l \in \Omega_{l n}} c_{l} w_{l}+\sum_{k \in \Omega_{g n}} c_{k} z_{k}+\sum_{k \in \Omega_{g}} c o_{k} g_{k i}+\sum_{i \in \Omega_{b}} D N A_{i} C_{D N A i}
$$

Sujeto a:

$$
\begin{aligned}
& \left(\sum_{l \in \Omega_{l e}} f_{l j i}+\sum_{l \in \Omega_{l n}} f_{l j i}\right)-\left(\sum_{l \in \Omega_{l e}} f_{l i j}+\sum_{l \in \Omega_{l n}} f_{l i j}\right)+\sum_{l \in \Omega_{g}} g_{k i}+D N A_{i}=d_{i} \quad \forall i \in \Omega_{b} \\
& \left(\sum_{l \in \Omega_{l e}} f_{l j i, c}+\sum_{l \in \Omega_{l n}} f_{l j i, c}\right)-\left(\sum_{l \in \Omega_{l e}} f_{l i j, c}+\sum_{l \in \Omega_{l n}} f_{l i j, c}\right)+\sum_{l \in \Omega_{g}} g_{k i} z_{k}+D N A_{i}=d_{i} \quad \forall i \in \Omega_{b}, \forall l \in \Omega_{c} \\
& f_{l i j}=S_{\text {base }} \frac{\left(\theta_{i}-\theta_{j}\right)}{x_{l}^{p u}} \\
& f_{l i j, c}=\left(1-\operatorname{CON}_{l, c}\right) S_{\text {base }} \frac{\left(\theta_{i, c}-\theta_{j, c}\right)}{x_{l}^{p u}} \\
& \frac{f_{\text {lij }} x_{l}^{p u}}{S_{\text {base }}}-\left(\theta_{i}-\theta_{j}\right) \leq 2 \bar{\theta}\left(1-w_{l}\right) \\
& \frac{f_{l i j} x_{l}^{p u}}{S_{\text {base }}}-\left(\theta_{i}-\theta_{j}\right) \geq-2 \bar{\theta}\left(1-w_{l}\right) \\
& \forall l \in \Omega_{l n} \\
& \frac{f_{l i j, c} x_{l}^{p u}}{S_{\text {base }}}-\left(\theta_{i, c}-\theta_{j, c}\right) \leq 2 \bar{\theta}\left(1-w_{l}\left(1-\operatorname{CON}_{l, c}\right)\right) \\
& \forall l \in \Omega_{l n}, \forall l \in \Omega_{c} \\
& \frac{f_{l i j, c} x_{l}^{p u}}{S_{\text {base }}}-\left(\theta_{i, c}-\theta_{j, c}\right) \geq-2 \bar{\theta}\left(1-w_{l}\left(1-\operatorname{CON}_{l, c}\right)\right) \\
& \forall l \in \Omega_{l n}, \forall l \in \Omega_{c} \\
& -\bar{f}_{l} \leq f_{l i j} \leq \bar{f}_{l} \\
& \forall l \in \Omega_{l e} \\
& \forall l \in \Omega_{l e}, \forall l \in \Omega_{c} \\
& \forall l \in \Omega_{l n} \\
& \forall l \in \Omega_{l e}
\end{aligned}
$$




$$
\begin{aligned}
& -\bar{f}_{l} w_{l} \leq f_{l i j} \leq \bar{f}_{l} w_{l} \\
& -\bar{f}_{l}\left(1-\operatorname{CON}_{l, c}\right) \leq f_{l i j, c} \leq \bar{f}_{l}\left(1-\operatorname{CON}_{l, c}\right) \\
& -\bar{f}_{l} w_{l}\left(1-\operatorname{CON}_{l, c}\right) \leq f_{l i j, c} \leq \bar{f}_{l} w_{l}\left(1-\operatorname{CON}_{l, c}\right) \\
& 0 \leq g_{k i} \leq \bar{g}_{k} \\
& 0 \leq g_{k i} \leq \bar{g}_{k} z_{k} \\
& -\bar{\theta} \leq \theta_{i} \leq \bar{\theta} \\
& -\bar{\theta} \leq \theta_{i, c} \leq \bar{\theta} \\
& w_{l} \text { binario; } \quad z_{k} \text { binario } \\
& \theta_{i}=0
\end{aligned}
$$

$$
\begin{aligned}
& \forall l \in \Omega_{l n} \\
& \forall l \in \Omega_{l e}, \forall l \in \Omega_{c} \\
& \forall l \in \Omega_{l n}, \forall l \in \Omega_{c} \\
& \forall k \in\left(\Omega_{g e}\right) \\
& \forall k \in\left(\Omega_{g n}\right) \\
& \forall i \in \Omega_{b} \\
& \forall i \in \Omega_{b} \\
& \forall l \in \Omega_{l n} ; \forall k \in \Omega_{g n} \\
& \forall i \in \Omega_{b} / i=r e f
\end{aligned}
$$

La ecuación (1) representa la función objetivo del modelo. En este caso se busca la minimización de los costos de inversión y operación. Los primeros dos términos representan los costos de inversión para nuevas líneas y generadores, respectivamente. Las variables binarias $z_{k}$ y $w_{l}$ se usan para indicar si existen nuevos generadores y líneas, respectivamente. El tercer término indica el costo de operación para generadores nuevos y existentes, donde la variable continua $g_{k i}$ representa la potencia suministrada por cada generador. Finalmente, el cuarto término representa el costo de la demanda no atendida.

Las restricciones (2) y (3) representan las ecuaciones de balance de potencia nodal en condición normal y bajo contingencia, respectivamente. Las ecuaciones (4) y (5) modelan los flujos de carga para las líneas existentes, la primera considerado red normal y la segunda considerando contingencias. Las restricciones (6) y (7) permiten calcular los flujos de carga para las líneas nuevas en condición normal, mientras que las restricciones dadas por las ecuaciones (8) y (9) representan los flujos de carga para las líneas nuevas bajo contingencia. Las restricciones dadas por las ecuaciones (10) y (11) limitan el flujo de potencia máximo de las líneas existentes y nuevas respectivamente en condición normal. Las restricciones (12) y (13) limitan el flujo de potencia máximo de las líneas existentes y nuevas, respectivamente bajo contingencia. La restricción dada por la ecuación (14) limita la generación máxima de cada generador existente, mientras que la restricción (15) limita la generación máxima de cada generador nuevo. Las restricciones (16) y (17) representan los límites máximos de los ángulos de las barras en condición normal y bajo contingencia, respectivamente. La restricción (18) indica la naturaleza binaria de las variables de decisión para la inversión en líneas y generadores. Finalmente, la ecuación (19) indica que el ángulo de la barra de referencia debe ser cero. Es importante aclarar que dado que el modelo es determinístico no es posible considerar los aspectos estocásticos inherentes a la producción de electricidad mediante algunas tecnologías de GD. Los resultados obtenidos con el modelo propuesto son indicativos en cuanto a la cantidad de generación a pequeña escala deseable en ciertos nodos de la red, para minimizar el costo de atender la demanda futura, cumpliendo con el criterio de seguridad $\mathrm{N}-1$.

\section{RESULTADOS}

Para verificar la validez del modelo propuesto se seleccionaron tres sistemas de prueba y diferentes escenarios de estudio. Los sistemas de prueba utilizados son el sistema Garver de 6 barras modificado, y los sistemas IEEE de 24 y 48 barras. Adicionalmente, se analizan dos escenarios de estudio para cada sistema de prueba. El escenario 1 considera costos altos en transmisión y el escenario 2 considera costos bajos en transmisión. Los resultados obtenidos en el sistema IEEE de 24 barras aplicando el modelo propuesto en este artículo se compararon con los resultados presentados en (Saldarriaga et al., 2016a). El modelo de PIEGT propuesto se formuló en GAMS y se solucionó mediante el optimizador CPLEX. A continuación, se presentan los resultados y análisis de los casos de prueba seleccionados.

\section{Sistema Garver de 6 barras}

Este sistema cuenta con 6 barras, 6 líneas, 2 generadores y 5 cargas que suman un valor de demanda de 190 MW. Se desea encontrar la inversión óptima de mínimo costo para su expansión cumpliendo con restricciones de seguridad $\mathrm{N}-1$. Para el escenario futuro a analizar se considera la conexión de una barra nueva al sistema, el valor de la carga futura es de 820 MW. Como candidatas de expansión en transmisión se consideraron todas las combinaciones posibles de corredores entre las 5 barras existentes y la barra nueva. 
En este sistema se permite adicionar hasta 2 líneas por cada corredor. Además, para cada nodo del sistema se consideró la posibilidad de instalar tres tipos de generadores como candidatos de expansión en generación. El generador tipo 1 tiene una potencia máxima de 10MW, el tipo 2 con20MW y el tipo 3 con 30MW. Como valor de inversión en generación se consideró un costo de 1 Millón\$/MW. Mientras que para el valor de inversión de líneas de transmisión se consideraron dos escenarios: precios altos y bajos. Para el escenario de precios de transmisión altos se consideraron los costos de líneas presentados en (Romero et al., 2005) y para el escenario de precios de transmisión bajos se consideraron los costos de líneas presentados en (Alizadeh y Jadid, 2011). En la Fig. 1 (a) y Fig. 1 (b) se presentan los planes encontrados para los escenarios de precios altos y bajos, respectivamente. En este caso, los elementos marcados en línea discontinua indican los nuevos elementos que propone el plan de expansión.

Se puede observar que los planes de expansión presentados en la Fig 1 proponen atender la carga de la barra 6 de forma local instalando generación en dicha barra. Para este plan no se propone conectar la barra 6 al sistema por medio de ninguna línea. Lo anterior es generado por los costos que se consideran en las líneas de transmisión para los escenarios y los criterios de seguridad considerados. Si solo hay una línea que atienda la barra 6 , la falla de esa línea generaría racionamiento. Se requeriría entonces, conectar dos o más líneas para atender la demanda de la barra 6 de forma segura, lo que incrementaría los costos. Por lo tanto, es más atractivo atender esta carga con generación en la barra 6 que instalar nuevas líneas de transmisión.

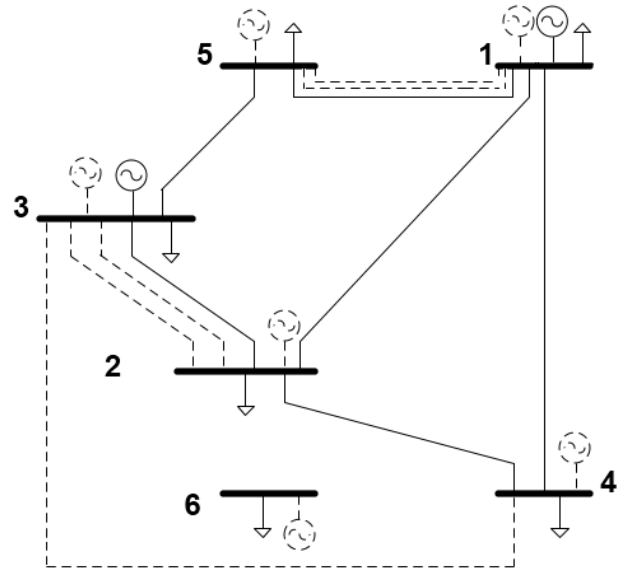

(a)

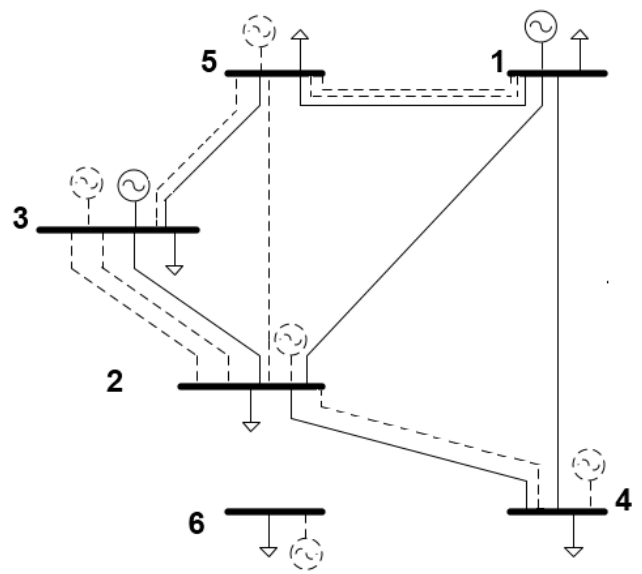

(b)

Fig. 1: Plan de expansión para el sistema Garver de 6 barras (a): escenario de precios altos en transmisión, (b): escenario de precios bajos en transmisión.

En la Tabla 1 se presenta el plan de expansión más económico que cumple las restricciones de seguridad N1, considerando un escenario de precios altos en los activos de transmisión. En la primera columna se presentan las líneas seleccionadas en el plan con sus nodos inicial y final. Por ejemplo, L1-5 indica la construcción de una línea en el corredor que va del nodo 1 al nodo 5 . En la segunda columna se indican los nuevos generadores. La etiqueta Nodo(MW) indica la ubicación y potencia del generador propuesto como solución. Por ejemplo, N1(30) significa que un generador de $30 \mathrm{MW}$ será instalado en el nodo 1. La solución encontrada recomienda 5 líneas y 8 generadores con una potencia instalada de 150MW. En la Tabla 2 se presenta el plan de expansión más económico que cumple las restricciones de seguridad $\mathrm{N}-1$, considerando un escenario de precios bajos en los activos de transmisión El plan recomienda 7 líneas y 7 generadores con una potencia instalada de $150 \mathrm{MW}$. En este caso, el plan considera 2 líneas más que el escenario de precios altos: un refuerzo en el corredor 2-4 y otro en el corredor 3-5.

Tabla 1: Plan de expansión para escenario de precios altos en activos de transmisión.

\begin{tabular}{|c|c|c|}
\hline Líneas de transmisión & Generadores Nodo (MW) & Costo ([M\$] ) \\
\hline L1-5, L1-5, L2-3, L2-3, L3-4 & $\begin{array}{l}\text { N1(10), N2(30), N3(30), N4(10), } \\
\text { N5(10), N6(10), N6(20), N6(30) }\end{array}$ & 289.057 \\
\hline
\end{tabular}

Tabla 2: Plan de expansión para escenario de precios bajos en activos de transmisión.

\begin{tabular}{|c|c|c|}
\hline Líneas de transmisión & Generadores (MW) & Costo ([M\$] ) \\
\hline $\begin{array}{c}\text { L1-5, L1-5, L2-3, L2-3, L2-4, } \\
\text { L2-5, L3-5 }\end{array}$ & $\begin{array}{c}\text { N2(20), N2(30), N4(10), N4(30), } \\
\text { N6(10), N6(20), N6(30) }\end{array}$ & 188.617 \\
\hline
\end{tabular}




\section{Sistema IEEE de 24 Barras}

Para evaluar el desempeño del modelo del PIEGT propuesto se realiza una comparación con el modelo propuesto en (Saldarriaga et al., 2016a). Para lo anterior se resolvió el problema en el sistema de prueba IEEE RTS-24 planteado en (Grigg et al., 1999). Este sistema cuenta con 24 barras, 38 líneas, 32 generadores y 17 cargas que suman un valor de demanda de $2850 \mathrm{MW}$. El valor de la carga futura es de $8550 \mathrm{MW}$, lo que representa tres veces el valor de la demanda actual. Para realizar las pruebas se consideraron los corredores existentes y 7 corredores nuevos como candidatas de expansión en transmisión. En este sistema se permite adicionar hasta 2 líneas por cada corredor. Los parámetros de los corredores considerados como candidatos pueden ser consultados en (Romero et al., 2005). Además, para cada nodo del sistema se consideró la posibilidad de instalar tres tipos de generadores como candidatos de expansión en generación similares a los considerados para el caso anterior.

En (Saldarriaga et al., 2016a) se propone un modelo multiobjetivo que busca minimizar el costo de inversión de la expansión y maximizar la seguridad del sistema ante contingencias simples. El último objetivo se obtiene minimizando las sobrecargas post-contingencia de las líneas en servicio. Esto se hace mediante la minimización del valor absoluto del máximo de los índices nodales ponderados de alivio de carga en transmisión (WTLR por sus siglas en inglés). Los índices WTLR permiten evaluar criterios de seguridad en los planes de expansión. El modelo propuesto en (Saldarriaga et al., 2016a) es solucionado por medio de un algoritmo genético multiobjetivo NSGA-II, el cual entrega un conjunto de soluciones que representan un compromiso entre costo y seguridad (frente óptimo de Pareto). Para comparar los resultados con el modelo propuesto en este artículo se toma una de las soluciones de (Saldarriaga et al., 2016a) que maximiza la seguridad, es decir, que cumple con el criterio N-1 (índices WTLR aproximadamente igual a cero).

En la Tabla y en la Tabla se comparan los resultados entre el modelo de PIEGT propuesto y el presentado en (Saldarriaga et al., 2016a) para los escenarios de precios altos y bajos en activos de transmisión, respectivamente. En el escenario de precios altos (Tabla 3) la solución encontrada por el modelo propuesto recomienda 22 líneas y 10 generadores con una potencia total instalada de $180 \mathrm{MW}$ y un costo de $1125.59 \mathrm{M} \$$. En la Fig. 2 se presenta este plan de expansión a manera de ilustración. Por otro lado, la solución reportada en (Saldarriaga et al., 2016a) recomienda 21 líneas y 11 generadores con una potencia total instalada de $190 \mathrm{MW}$ y un costo de $1270 \mathrm{M} \$$. En contraste, si no se tiene en cuenta la participación de generación en el modelo se necesitarían 36 líneas con un costo total de 1806M\$. Esto último evidencia el impacto de considerar generación en la planeación.

Tabla 3: Plan de expansión escenario de precios altos en activos de transmisión

\begin{tabular}{|c|c|c|c|}
\hline Líneas de transmisión & Generadores Nodo (MW) & $\begin{array}{l}\text { Costo } \\
([M \$])\end{array}$ & Modelo \\
\hline $\begin{array}{l}\text { L1-5, L3-9, L3-9, L3-24, L3-24, L4-9, L6-10, L6- } \\
\text { 10, L7-8, L7-8, L10-11, L10-12, L13-14, L14-23, } \\
\text { L14-23, L15-21, L15-24, L15-24, L16-17, L16- } \\
\text { 17, L16-19, L17-18 }\end{array}$ & $\begin{array}{l}\text { N5(10), N5(20), N6(10), N6(20), } \\
\text { N6(30), N10(10) N10(20), } \\
\text { N11(10), N11(20), N11(30) }\end{array}$ & 1125.59 & PIEGT Propuesto \\
\hline $\begin{array}{l}\text { L3-24, L6-10, L9-11, L9-12, L14-16, L16-17, } \\
\text { L17-18, L17-22, L1-8, L2-4, L4-9, L8-9, L10-11, } \\
\text { L11-14, L13-23, L14-16, L15-21, L15-24, L16- } \\
\text { 17, L16-19, L20-23 }\end{array}$ & $\begin{array}{l}\text { N3(20), N5(10), N11(10), } \\
\text { N11(30), N12(30), N13(30), } \\
\text { N16(10), N17(10), N22(10), } \\
\text { N23(10), N24(20), }\end{array}$ & 1270 & $\begin{array}{l}\text { PIEGT } \\
\text { (Saldarriaga et al., } \\
\text { 2016a). }\end{array}$ \\
\hline
\end{tabular}

En el escenario de precios bajos en transmisión (Tabla 4) la solución encontrada por el método propuesto en este artículo recomienda 25 líneas y 10 generadores con una potencia instalada de 190MW con un costo total de $866.668 \mathrm{M} \$$. Por otro lado, la solución reportada en (Saldarriaga et al., 2016a) recomienda la construcción de 28 líneas y 10 generadores con una potencia instalada de $150 \mathrm{MW}$ y un costo total de $892 \mathrm{M} \$$. Se evidencia que en ambos casos (precios altos y bajos en transmisión) el modelo propuesto pudo encontrar soluciones más económicas que las encontradas por el algoritmo NSGA-II. Esto ocurre porque las metaheurístias en general, no pueden garantizar optimalidad en las soluciones mientras la solución de un modelo de programación lineal entera mixta por métodos convencionales si la garantiza. Por otro lado, la ventaja del NSGA-II radica en la obtención de un conjunto de soluciones que representan un compromiso en entre varios objetivos en lugar de una sola solución con un único objetivo. 
Tabla 4. Plan de expansión escenario de precios bajos en activos de transmisión

\begin{tabular}{|c|c|c|c|}
\hline Líneas de transmisión & Generadores (MW) & Costo $([M \$])$ & Modelo \\
\hline $\begin{array}{l}\text { L1-5,L1-5,L3-9,L3-24,L3-24,L5-10,L6-10,L6- } \\
\text { 10,L7-8,L7-8,L9-12,L10-11,L10-12,L12- } \\
\text { 23,L13-23,L14-16,L14-16,L15-24,L16-17,L16- } \\
\text { 17,L17-18,L17-18,L18-21,L18-21,L20-23 }\end{array}$ & $\begin{array}{l}\text { N3(10), N4(20), N4(30), } \\
\text { N5(20), N9(10), N15(10), } \\
\text { N15(30), N24(10), N24(20), } \\
\text { N24(30) }\end{array}$ & 866.66 & PIEGT Propuesto \\
\hline $\begin{array}{l}\text { L2-6, L3-24, L4-9, L5-10, L6-10, L7-8, L8-9, } \\
\text { L9-11, L10-12, L14-16, L15-21, L21-22, L14- } \\
\text { 23, L1-2, L1-3, L1-5, L3-9, L3-24, L6-10, L11- } \\
\text { 14, L15-21, L15-24, L16-17, L17-18, L18-21, } \\
\text { L20-23, L1-8, L13-14 }\end{array}$ & $\begin{array}{l}\text { N2(10), N3(20), N4(30), } \\
\text { N5(20), N6(10), N7(20), } \\
\text { N9(10), N18(10), N20(10), } \\
\text { N22(10) }\end{array}$ & 892 & $\begin{array}{l}\text { PIEGT (Saldarriaga } \\
\text { et al., 2016a). }\end{array}$ \\
\hline
\end{tabular}

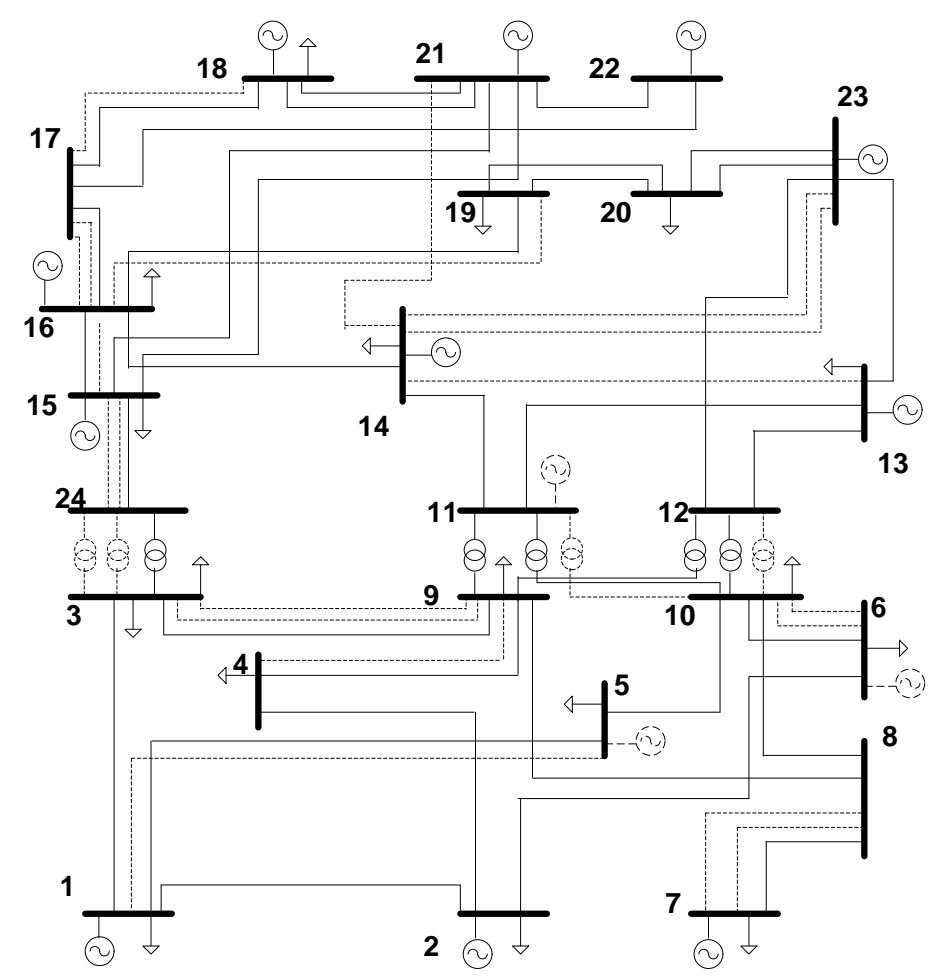

Fig. 2: Plan de expansión para el sistema IEEE de 24 barras considerando un escenario de precios altos en transmisión.

\section{Sistema IEEE de 48 Barras}

Este sistema consta de 48 barras, 78 ramas y debe suplir una demanda futura de $17100 \mathrm{MW}$. Los datos del sistema IEEE de 48 barras pueden ser consultados en (Grigg et al., 1999). En este sistema se permite adicionar hasta 2 líneas por cada corredor. Los parámetros de los corredores considerados como candidatos pueden ser consultados en (Romero et al., 2005). De forma similar al caso anterior se ejecuta el modelo propuesto para el PIEGT considerando escenarios de precios altos y bajos en transmisión. En la Tabla 5 se presentan los resultados obtenidos. En este caso no se cuenta con resultados reportados en otros trabajos que puedan ser comparables. En la Tabla 5 se puede observar que al considerar un escenario de precios altos en transmisión la solución del PIEGT considera 44 nuevos circuitos y la instalación de 360MW en generación para un costo total de $2251.18 \mathrm{M} \$$. Por otro lado, cuando se consideran precios bajos en transmisión la solución para el PIEGT considera 50 nuevos circuitos y 380MW en generación. En ambos casos la solución garantiza la seguridad del sistema ante contingencias $\mathrm{N}-1$, siendo la diferencia en precios de ambas soluciones debida al costo de los activos de transmisión. En la Fig. 3 se ilustra la solución encontrada considerando el escenario de precios altos en transmisión. Los nuevos elementos (líneas y generadores) se representan mediante línea punteada. 
Tabla 5: Plan de expansión sistema IEEE 48 barras

\begin{tabular}{|c|c|c|c|}
\hline Precios Transmisión & Líneas de transmisión & Generadores Nodo (MW) & $\begin{array}{l}\text { Costo } \\
([\mathrm{M} \$])\end{array}$ \\
\hline Altos & $\begin{array}{l}\text { L1-5, L3-9, L3-9, L3-24, L3-24, L4-9, L6-10, } \\
\text { L6-10, L7-8, L7-8, L10-11, L10-12, L13-14, } \\
\text { L14-23, L14-23, L15-21, L15-24, L15-24, } \\
\text { L16-17, L16-17, L16-19, L17-18, L25-29, } \\
\text { L27-23, L27-23, L27-48, L27-48, L28-33, } \\
\text { L30-34, L30-34, L31-32, L31-32, L34-35, } \\
\text { L34-36, L37-38, L38-47, L38-47, L39-45, } \\
\text { L39-48, L39-48, L40-41, L40-41, L40-43, } \\
\text { L41-42 }\end{array}$ & $\begin{array}{c}\text { N5(10), N5(20), N6(10), } \\
\text { N6(20), N6(30), N10(10) } \\
\text { N10(20), N11(10), N11(20), } \\
\text { N11(30), N29(10), N29(20), } \\
\text { N30(10), N30(20), N31(30), } \\
\text { N34(10), N34(20), N35(10), } \\
\text { N35(20), N35(30) }\end{array}$ & 2251.18 \\
\hline Bajos & $\begin{array}{l}\text { L1-5, L1-5, L3-9, L3-24, L3-24, L5-10, L6-10, } \\
\text { L6-10, L7-8, L7-8, L9-12, L10-11, L10-12, } \\
\text { L12-23, L13-23, L14-16, L14-16, L15-24, } \\
\text { L16-17, L16-17, L17-18, L17-18, L18-21, } \\
\text { L18-21, L20-23, L25-29, L25-29, L27-33, } \\
\text { L27-48, L27-48, L29-34, L30-34, L30-34, } \\
\text { L31-32, L31-32, L33-36, L34-35, L34-36, } \\
\text { L36-47, L37-47, L38-40, L38-40, L39-48, } \\
\text { L40-41, L40-41, L41-42, L41-42, L42-44, } \\
\text { L42-45, L44-47 }\end{array}$ & $\begin{array}{l}\text { N3(10), N4(20), N4(30), } \\
\text { N5(20), N9(10), N15(10), } \\
\text { N15(30), N24(10), N24(20), } \\
\text { N24(30), N27(10), N28(20), } \\
\text { N28(30), N29(20), N33(10), } \\
\text { N39(10), N39(30), N48(10), } \\
\text { N48(20), N48(30), }\end{array}$ & 1733.33 \\
\hline
\end{tabular}

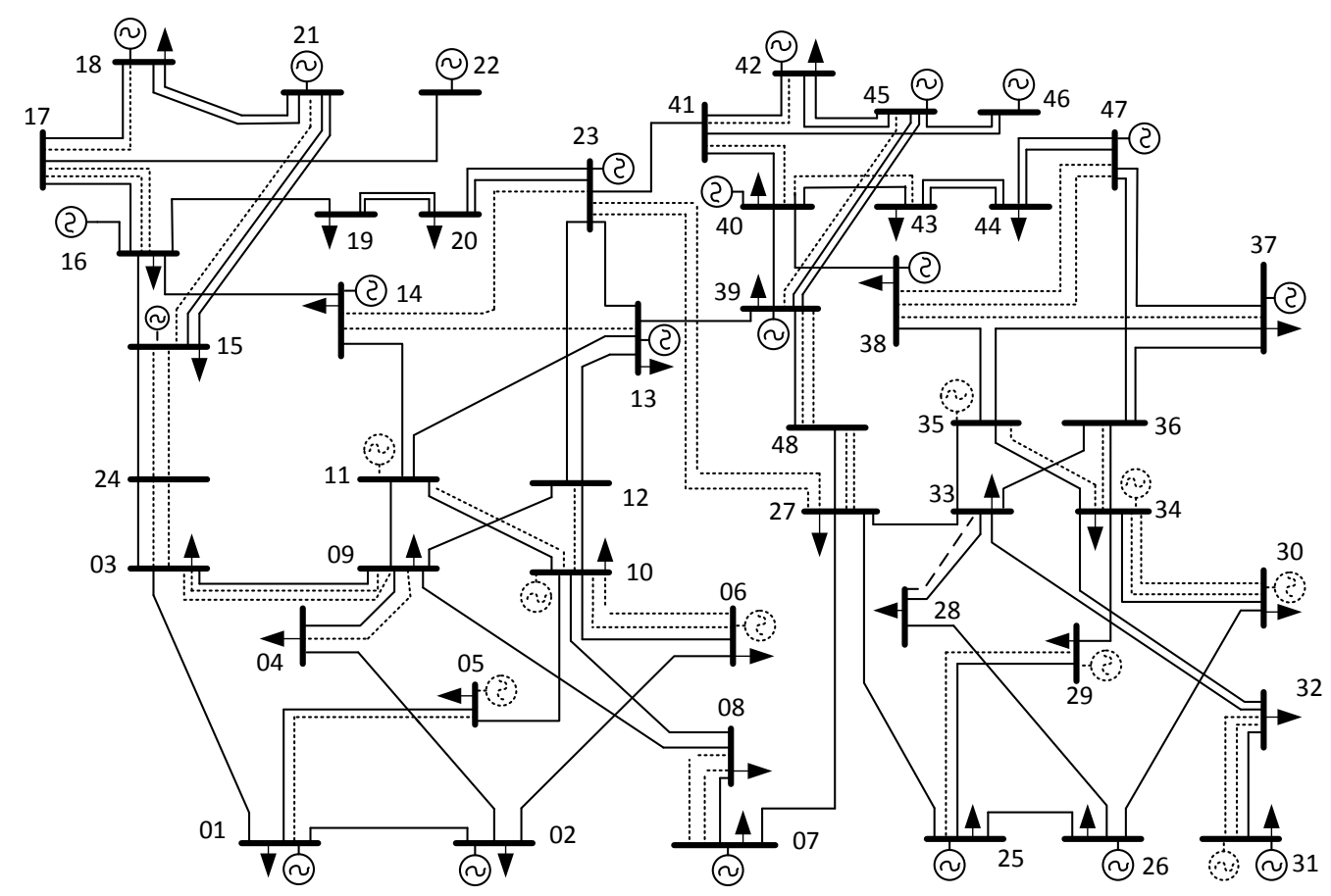

Fig. 3: Plan de expansión para el sistema IEEE de 48 barras considerando un escenario de precios altos en transmisión.

\section{CONCLUSIONES}

En este artículo se presentó un modelo que combina, en un único problema de optimización, el planeamiento de la expansión en transmisión con el planeamiento de la expansión en generación. El modelo de optimización propuesto es abordado mediante programación lineal entera mixta, lo que garantiza la optimalidad de las soluciones encontradas. La principal contribución del trabajo consiste en la incorporación de restricciones de seguridad en este tipo de modelos (criterio N-1). Los resultados obtenidos en el sistema Garver de 6 barras y los sistemas IEEE de 24 y 48 barras evidencian la efectividad y aplicabilidad del modelo. En las pruebas se consideraron escenarios de precios altos y bajos en activos de transmisión. Se pudo verificar que considerar generación a pequeña como componentes de los candidatos de solución permite reducir de forma importante el costo del plan de expansión, pues el número de líneas requerido es menor. Este fenómeno se observa especialmente cuando se consideran costos altos en los activos de transmisión. En un trabajo futuro se planea considerar el efecto de la incertidumbre en el modelo PIEGT para incluir el impacto de la incorporación de energías renovables en el planeamiento. 


\section{NOTACIÓN}

Conjuntos:

$\Omega_{b}$ : Conjunto de barras.

$\Omega_{l}$ : Conjunto de líneas.

$\Omega_{g}$ : Conjunto de generadores.

$\Omega_{l n}$ : Conjunto de líneas nuevas.

$\Omega_{g n}$ : Conjunto de generadores nuevos.

$\Omega_{l e}$ : Conjunto de líneas existentes.

$\Omega_{g e}$ : Conjunto de generadores existentes.

$\Omega_{c}$ : Conjunto de contingencias.

Parámetros:

$d_{i}$ : Demanda en la barra $i[\mathrm{MW}]$.

$\bar{g}_{k}$ : Generación máxima del generador $k[\mathrm{MW}]$.

$c_{l}$ : Costo de inversión de la línea $l[\$]$.

$c_{k}$ : Costo de inversión del generador $k[\$]$.

$c o_{k}$ : Costo de operación del generador $k[\$ / \mathrm{MW}]$.

$\bar{f}_{l}$ : Flujo de potencia máximo en la línea $l[\mathrm{MW}]$.

$x_{l}^{p u}:$ Reactancia de la línea $l[p . u]$.

$S_{\text {base }}:$ Potencia Base [MW].

$\bar{\theta}$ : Ángulo de fase máximo [rad].

$C_{D N A}$ : Costo de la demanda no atendida [\$/MW].

$\operatorname{CON}_{l, c}$ : Matriz dispersa de 1 y 0 que representa cada elemento bajo contingencia

\section{Variables:}

$w_{l}$ : Línea nueva $l$.

$z_{k}$ : Generador nuevo $k$.

$D N A_{i}$ : Demanda no atendida en el nodo $i[\mathrm{MW}]$.

$g_{k i}$ : Generación de potencia activa del generador $k$ conectado al nodo $i[\mathrm{MW}]$.

$\theta_{i}$ : Ángulo de fase en la barra $i[\mathrm{rad}]$.

$\theta_{i, c}$ : Ángulo de fase en la barra $i$ ante la contingencia $c$ [rad].

$f_{l i j}$ : Flujo de potencia por la línea $l$ conectada entre los nodos $i, j$ en condición normal de operación [MW].

$f_{l i j, c}$ : Flujo de potencia por la línea $l$ conectada entre los nodos $i, j$ ante la contingencia $c$ [MW].

\section{AGRADECIMIENTOS}

Los autores agradecen a la Universidad de Antioquia (UdeA) por el apoyo del programa de sostenibilidad.

\section{REFERENCIAS}

Akbari, T.; A. Rahimikian y A. Kazemi, A multi-stage stochastic transmission expansion planning method, Energy conversion and Management, 52(8), 2844-2853 (2011)

Alizadeh, B. y S. Jadid, Reliability constrained coordination of generation and transmission expansion planning in power systems using mixed integer programming, IET Generation, Transmission \& Distribution, 5(9), 948-960 (2011)

Benidris, M.; S. Elsaiah y J. Mitra, An emission-constrained approach to power system expansion planning, International Journal of Electrical Power \& Energy Systems, 85(1), 78-86 (2016)

Bent, R. y L. Toole, Grid expansion planning for carbon emissions reduction, IEEE Power and Energy Society General Meeting, San Diego-California, July $22^{\text {th }}-26^{\text {th }}(2012)$

Cerda, P.; M. Larrain y H. Rudnick, Identification of generation expansion plans in competitive markets, IEEE Latin America Transactions, 9(5), 774-784 (2011)

Gomes, P.V. y J.T. Saraiva, Hybrid discrete evolutionay PSO for AC dynamic transmission expansion planning IEEE International Energy Conference Leuven-Belgium, April $4^{\text {th }}-8^{\text {th }},(2016)$

Grigg, C.; P. Wong; P. Albrecht; R. Allan; M. Bhavaraju; R. Billinton; Q. Chen; C. Fong; S. Haddad; S. Kuruganty; W. Li, R. Mukerji, D. Patton; N. Rau; D. Reppen; A. Schneider; M. Shahidehpour y C. Singh, The IEEE reliability test system 1996 a report prepared by the reliability test system task force of the application of probability methods subcommittee, IEEE Transactions on Power systems, 14(3), 1010-1020 (1999)

Hemmati, R. y R.A. Hooshmand, State-of-the-art of transmission expansion planning : Comprehensive review, Renewable and Sustainable Energy Reviews, 23(1), 312-319 (2013) 
Hobbs B.F.; Q. Xu; J. Ho; P. Donohoo y S. Kasina, Adaptive transmission planning: implementing a new paradigm for managing economic risks in grid expansion, IEEE Power and Energy Magazine, 14(4), 30-40 (2016)

Li, C.; Z. Dong; Y. Chen; G. Luo y F. Liu, Flexible transmission expansion planning associataed with large-scale wind farms integration considering demand response, IET Generation Transmission and Distribution, 10(10), 2351-2359 (2016)

Macedo C.V.; J.F. Franco; M.J. Rider y R. Romero, MILP branch flow model for concurrent AC multistage transmission expansion and reactive power planning with security constraints, IET Generation Transmission and Distribution, 10(12), 3023-3032 (2016)

Majidi, Q.M. y R. Baldick, Integration of $\mathrm{N}-1$ contingency analysis with systematic transmission capacity expansion planning: ERCOT case study, IEEE Transactions on Power Systems, 31(3), 2234-2245 (2016)

Murugan P.; S. Kanna y S. Baskar, NSGA-II Algorithm for multi-objective generation expansion problem, Electric Power System Research, 79(4), 622-628 (2009)

Phupha, V y T. Lantharthong, Generation Expansion Planning Strategies on Power System : A Review, World Academy of Science Engineering and Tecnology, 6(4), 436-439 (2012)

Pozo, D.; E. Sauma, y J. Contreras, A Three-Level Static MILP Model for Generation and Transmission Expansion Planning, IEEE Transactions on Power systems, 28(1), 202-210 (2013)

Quintero, J.; H. Zhang; Y. Chakhchoukh; V. Vittal, y G.T. Heydt, Next generation transmission expansion planning framework: models, tools, and educational opportunities, IEEE Transactions on Power systems, 29(4), 911-1918, (2014)

Ramírez, P.; D. Papadaskalopoulos y G. Strba, Co-optimization of generation expansion planning and electric vehicles flexibility, IEEE Transactions on Smart Grids, 7(3), 1609-1619 (2016).

Romero, R.; C. Rocha; J.R. Mantovani, y I.G. Sanchez, Constructive heuristic algorithm for the DC model in network transmission expansion planning, IEE Proceedings on Generation, Transmission and Distribution:

152(2) 277-281 (2005)

Rouhani, A.; S.H. Hosseini y M. Raoofat, Composite generation and transmission expansion planning considering distributed generation, International Journal of Electrical Power \& Energy Systems, 62(1), 792-805 (2014)

Saboori, H. y H. Reza, Considering carbon capture and storage in electricity generation planning, IEEE Transactions on Sustainable Energy, 7(4), 1371-1378 (2016)

Saldarriaga Z.S.D.; J.M López-Lezama y N. Muñoz-Galeano, Planeamiento de la expansión generación-transmisión utilizando los índices nodales ponderados de alivio de carga en transmisión (WTLR), Información tecnológica, 27(6), 193$202(2016 a)$

Saldarriaga Z.S.D.; J.M López-Lezama y N. Muñoz-Galeano, Planeamiento de la expansión integrada generacióntransmisión: una revisión del estado del arte, Tecnológicas, 19(37), 79-92 (2016b)

Tejada-Arango, D.; J.M. López-Lezama y M. Rider, Planeamiento de la expansion de la red de transmisión en sistemas eléctricos de potencia considerando reconfiguraciones de red, Información Tecnológica, 25(1), 85-96 (2014)

Tekiner H.; D.W. Cobit y F.A.Felder, Multi-period multi-objective electricity generation expansion planning problem with Monte-Carlo Simulation, Electric Poser System Research, 80(12), 1394-1405 (2010) 